\title{
The systemic inflammatory response as a prognostic factor for advanced hepatocellular carcinoma with extrahepatic metastasis
}

\author{
HAJIME AINO, SHUJI SUMIE, TAKASHI NIIZEKI, RYOKO KUROMATSU, NOBUYOSHI TAJIRI, \\ MASAHITO NAKANO, MANABU SATANI, SHUSUKE OKAMURA, SHIGEO SHIMOSE, \\ KENSUKE MIYAHARA and TAKUJI TORIMURA
}

Division of Gastroenterology, Department of Medicine, Kurume University School of Medicine, Kurume, Fukuoka 830-0011, Japan

Received October 6, 2015; Accepted January 26, 2016

DOI: $10.3892 / \mathrm{mco} .2016 .879$

\begin{abstract}
Several indices have been proposed to evaluate the systemic inflammatory response (SIR), which has been reported to be a useful prognostic factor in various types of cancer. We investigated the usefulness of the Glasgow Prognostic Score (GPS), neutrophil-to-lymphocyte ratio (NLR) and platelet-to-lymphocyte ratio (PLR) as prognostic factors in patients with advanced hepatocellular carcinoma (HCC) with extrahepatic metastasis (stage IVB). Between April, 1997 and March, 2013, a total of 434 HCC patients who developed extrahepatic metastasis were enrolled in the present study. The GPS was defined on the basis of pretreatment $\mathrm{C}$-reactive protein (CRP) and albumin (Alb) levels, and the subjects were grouped according to GPS 0-2. The NLR was calculated as the neutrophil count/lymphocyte count, and the PLR was calculated as the platelet count/lymphocyte count. A comparative examination was performed using a survival analysis with approximate median values to determine the cut-off value for both ratios. The median survival time (MST) of the 434 patients overall was 7.3 months, with cumulative survival rates of $31.8,14.5$ and $7.7 \%$ at 1,2 and 3 years, respectively. The patient backround was as follows: The male:female ratio was $363: 71$, with a median age of 67.0 years (range, 15.0-92.0 years). Hepatitis B virus patients:hepatitis $C$ virus patients:non- $B$, non-C hepatitis patients $=75: 303: 56$. Child-Pugh class A:B:C $=218: 153: 63$. As regards T stage, $\leq \mathrm{T} 2: \mathrm{T} 3: \mathrm{T} 4=60: 190: 181$. The median white blood cell count was 4,650/1 (range, 1,400-20,500/1); the platelet count was $11.1 \times 10^{4} / \mu 1$ (range, $3.1 \times 10^{4}-45.5 \times 10^{4} / \mu 1$ ); the aspartate aminotransferase level was $40.0 \mathrm{U} / 1$ (range, 7.0-338.0 U/1) and the alanine aminotransferase level $64.5 \mathrm{U} / 1$ (range, 16.0-407.0 U/l); the $\alpha$-fetoprotein level was $622.1 \mathrm{ng} / \mathrm{ml}$ (range, 1.5-3,311,794.0 $\mathrm{ng} / \mathrm{ml}$ ); and the
\end{abstract}

Correspondence to: Dr Hajime Aino, Division of Gastroenterology, Department of Medicine, Kurume University School of Medicine, 67 Asahi-machi, Kurume, Fukuoka 830-0011, Japan

E-mail: aino0217@kph.biglobe.ne.jp

Key words: hepatocellular carcinoma, extrahepatic metastasis, systemic inflammatory response des-gamma-carboxyprothrombin level was $1,285.0 \mathrm{mAU} / \mathrm{ml}$ (range, 8.0->75,000 mAU/ml). The principal sites of metastasis included the lungs $(53.9 \%)$, bone $(38.9 \%)$, lymph nodes $(21.4 \%)$ and adrenal glands (10.1\%). The survival analysis revealed that hepatic functional reserve [Child-Pugh class $\mathrm{B}+\mathrm{C}$; hazard ratio $(\mathrm{HR})=2.055 ; 95 \%$ confidence interval $(\mathrm{CI}): 1.592-2.651$, $\mathrm{P}<0.001]$, T stage $(\mathrm{T} 3 ; \mathrm{HR}=2.359 ; 95 \% \mathrm{CI}: 1.648-3.376$, $\mathrm{P}<0.001)$, AFP $(\geq 200 \mathrm{ng} / \mathrm{ml}$; HR=1.416; 95\% CI: $1.125-1.783$, $\mathrm{P}=0.003), \mathrm{NLR}(\geq 3$; HR=1.569; 95\% CI: 1.253-1.963, $\mathrm{P}<0.001)$ and GPS $(1+2 ; \mathrm{HR}=1.410 ; 95 \% \mathrm{CI}: 1.060-1.874, \mathrm{P}=0.018)$ were independent risk factors. A total of 136 patients were included in the GPS 0 group, 169 patients in the GPS 1 group and 129 patients in the GPS 2 group. The low together with the high NLR groups comprised 217 patients. The MST was 480 days in the GPS 0 group, 154 days in the GPS 1 and 2 groups, 115 days in the high NLR group and 321 days in the low NLR group; a significant difference in survival was observed for the GPS and NLR groups. Therefore, we consider GPS and NLR to be useful prognostic factors in patients with stage IVB HCC.

\section{Introduction}

Hepatocellular carcinoma (HCC) is reported to be the sixth most common malignancy worldwide, affecting 626,000 individuals annually. In terms of mortality rate, $\mathrm{HCC}$ is among the malignancies with the poorest prognosis worldwide, coming third after lung cancer and gastric cancer (1). HCC has a high rate of recurrence, which shortens long-term survival, with extrahepatic metastasis known to develop during the course of the disease $(2,3)$. While there has been no effective treatment established for advanced HCC with extrahepatic metastasis (stage IVB), as of 2008, two placebo-controlled randomized trials have been underway. These trials have demonstrated the usefulness of the multikinase inhibitor sorafenib in treating advanced HCC patients with extrahepatic metastasis $(4,5)$. This has led to the recommendation of the use of sorafenib as a standard treatment for advanced HCC in Japan (6,7). However, while sorafenib prolongs survival, it is insufficient; therefore, a new agent or combined treatment is required for the treatment of patients with stage IVB HCC. It is important to elucidate the clinical characteristics and prognostic factors of such patients, in order to identify optimal treatment methods. The usefulness 
of inflammatory markers, such as the systemic inflammatory response (SIR), as prognostic factors of cancer has been recently reported (8). The usefulness of the neutrophil-to-lymphocyte ratio (NLR) as a predictive factor following treatment for HCC [transplantation, resection, radiofrequency ablation (RFA), and transcatheter arterial chemoembolization (TACE)], has also been reported (9-13). In general, cancer-related inflammatory responses affect cellular proliferation, cell survival, angiogenesis, tumor cell migration, invasion, metastasis and inhibition of adaptive immunity, indicating that cancer and inflammation are closely associated (14). Cancer may develop on a background of inflammation, such as chronic hepatitis, chronic gastritis, chronic pancreatitis and chronic inflammatory bowel disease. By contrast, cancer may also activate transcription factors and lead to systemic or localized inflammation mediated by the main inflammatory cytokines. Cancer and the inflammatory response have a strong involvement in their respective reciprocal development (15). An underlying cause of HCC is continuous infection with the hepatitis virus, i.e., it is strongly associated with inflammation. Therefore, the measurement of inflammatory markers should be clinically useful. The aim of the present study was to investigate the usefulness of the SIR as a prognostic factor for stage IVB HCC.

\section{Patients and methods}

Patient characteristics. Between April, 1997 and March, 2013, a total of 434 patients diagnosed with stage IVB HCC at Kurume University Hospital were enrolled in the present study. Hepatic functional reserve was determined using the Child-Pugh classification system and tumor staging was performed in accordance with the sixth edition of the American Joint Committee on Cancer/Union for International Cancer Control tumor-node-metastasis classification (16). The characteristics of the 434 patients are presented in Table I.

The sample comprised 363 male (83.6\%) and 71 female patients, with a median age of 67.0 years (range, $15-92$ years). Of the 434 patients, $303(69.8 \%)$ were positive for hepatitis C virus and 75 (17.3\%) were positive for hepatitis B virus. A total of 218, 153 and 63 patients were Child-Pugh class A, B and C, respectively. Following initial tumor staging, 60, 190 and 181 patients were classified as T0-2, T3 and T4, respectively. The sites of extrahepatic metastasis included the lungs in 234 patients (53.9\%), bone in 169 (38.9\%), lymph nodes in $93(21.4 \%)$, adrenal glands in $44(10.1 \%)$, the peritoneum and/or pleura in 31 (7.1\%), diaphragm in $21(4.8 \%)$, brain in $1(2.5 \%)$ and other sites in 69 patients (15.9\%).The treatment for stage IVB HCC patients $(n=434)$ included hepatectomy in $12(2.8 \%)$, percutaneous ethanol injections and RFA in $8(1.8 \%)$, TACE in 114 (26.3\%), hepatic arterial infusion chemotherapy in $216(49.8 \%)$, systemic chemotherapy in $69(15.9 \%)$, and radiation therapy in 127 patients $(29.3 \%)$. A total of 50 patients $(11.5 \%)$ did not fall under the aforementioned treatments categories; for these patients, treatment was administered as a combined multimodal approach, including systematic treatments that each patient was able to receive continuously.

Blood parameters. Routine laboratory measurements of haemoglobin, white cell count (WBC), albumin (Alb) and C-reactive protein (CRP) concentration were performed. The coefficient
Table I. Clinicobiochemical characteristics in the $434 \mathrm{HCC}$ patients with extrahepatic metastases $(n=434)$.

\begin{tabular}{lc}
\hline Characteristics & Values \\
\hline Gender (male/female) & $363 / 71$ \\
Age, years & $67.0(15.0-92.0)$ \\
Eiology (HCV/HBV/others) & $303 / 75 / 56$ \\
Child-Pugh class (A/B/C) & $218 / 153 / 63$ \\
Albumin, g/dl & $3.3(2.0-4.8)$ \\
AST, U/l & $40.0(7.0-338.0)$ \\
CRP, mg/dl & $0.50(0.0-16.58)$ \\
WBC count, x10 $/ 1$ r & $4.6(1.4-20.5)$ \\
Neutrophil count $/ \mu 1$ & $2,916(522-10,413)$ \\
Lymphocyte count/ $/ \mu 1$ & $967(98-3,749)$ \\
Platelet count, x10 $/ 1$ & $111(31-678)$ \\
AFP, ng/ml & $622.1(1.5-3,311,794.0)$ \\
DCP, mAU/ml & $1285.0(8.0-75,000.0)$ \\
Primary tumor stage ${ }^{a}(T 0-2 / T 3 / T 4)$ & $60 / 190 / 181$ \\
Site of extrahepatic metastasis, $\mathrm{n}(\%)$ & \\
Lungs & $234(53.9)$ \\
Bones & $169(38.9)$ \\
Lymph nodes & $93(21.4)$ \\
Adrenal glands & $44(10.1)$ \\
\hline
\end{tabular}

${ }^{\text {aT }}$ Tumor-node-metastasis classification. Continuous variables are presented as median (range). HCC, hepatocellular carcinoma; $\mathrm{HCV}$, hepatitis $\mathrm{C}$ virus; $\mathrm{HBV}$, hepatitis B virus; AST, aspartate aminotransferase; CRP, C-reactive protein; WBC, white blood cell; NLR, neutrophil-to-lymphocyte ratio; AFP, $\alpha$-fetoprotein; DCP, des-gamma-carboxyprothrombin.

of variation for these methods over the range of measurement was $<5 \%$, as established by routine quality control procedures.

$N L R-P L R$. The value measured on the day of diagnosis was used for each measurement. The NLR was calculated as the absolute neutrophil count/absolute lymphocyte count, whereas the PLR was calculated as the absolute platelet count/absolute lymphocyte count, and the median value of each was used for the respective cut-off value. The patients were then divided into two groups and the overall survival time for each factor was comparatively examined using Cox proportional hazard analysis. The median values for the NLR and PLR were 3.02 and 111.37, respectively. Analyses were performed using the approximate cut-off values of 3 for NLR and 111 for PLR.

Glasgow prognostic score (GPS). Based on pretreatment CRP and Alb levels, GPS was defined as 0 (CRP $\leq 1.0 \mathrm{mg} / \mathrm{dl}$ and $\mathrm{Alb} \geq 3.5 \mathrm{mg} / \mathrm{dl}), 1$ (CRP $>1.0 \mathrm{mg} / \mathrm{dl}$ and $\mathrm{Alb}<3.5 \mathrm{~g} / \mathrm{dl})$ and 2 (CRP $>1.0 \mathrm{mg} / \mathrm{dl}$ and Alb $<3.5 \mathrm{mg} / \mathrm{dl})$. Each patient was scored between 0 and 2 and the patients were divided into two groups, namely GPS $0-1$ and 2 . The overall survival times were comparatively examined using the Cox proportional hazard analysis. 
Table II. Univariate and multivariate analysis of survival in all $434 \mathrm{HCC}$ patients with extrahepatic metastasis.

\begin{tabular}{|c|c|c|c|c|}
\hline \multirow[b]{2}{*}{ Variables } & \multicolumn{2}{|c|}{ Univariate analysis } & \multicolumn{2}{|c|}{ Multivariate analysis } \\
\hline & HR $(95 \%$ CI $)$ & P-value & HR $(95 \%$ CI $)$ & P-value \\
\hline Gender (male) & $0.95(0.71-1.27)$ & 0.741 & & \\
\hline Age ( $\geq 65$ years) & $1.06(0.86-1.32)$ & 0.578 & & \\
\hline Etiology (HCV infection) & $0.97(0.77-1.22)$ & 0.807 & & \\
\hline Child-Pugh class $(B+C)$ & $2.76(2.21-3.44)$ & $<0.001$ & $2.03(1.58-2.60)$ & $<0.001$ \\
\hline $\operatorname{AST}(\geq 80 \mathrm{U} / \mathrm{l})$ & $1.18(0.90-1.55)$ & 0.241 & & \\
\hline WBC count $\left(\geq 6.0 \times 10^{9} / 1\right)$ & $1.78(1.41-2.26)$ & $<0.001$ & & \\
\hline NLR $(\geq 3.0)$ & $1.96(1.58-2.43)$ & $<0.001$ & $1.46(1.16-1.85)$ & 0.002 \\
\hline Platelet count $\left(\geq 120 \times 10^{9} / 1\right)$ & $0.94(0.76-1.17)$ & 0.579 & & \\
\hline $\operatorname{PLR}(\geq 111.0)$ & $1.38(1.11-1.71)$ & 0.004 & & \\
\hline GPS $(\geq 1)$ & $2.61(2.04-3.33)$ & $<0.001$ & $1.30(1.10-1.54)$ & 0.003 \\
\hline $\operatorname{AFP}(\geq 200$ ng/ml $)$ & $1.81(1.45-2.26)$ & $<0.001$ & $1.39(1.10-1.76)$ & 0.005 \\
\hline $\mathrm{DCP}(\geq 200 \mathrm{mAU} / \mathrm{ml})$ & $1.10(0.99-1.23)$ & 0.066 & & \\
\hline Primary tumor stage ${ }^{\mathrm{a}}(\geq \mathrm{T} 3)$ & $2.88(2.03-4.10)$ & $<0.001$ & $2.25(1.56-3.23)$ & $<0.001$ \\
\hline \multicolumn{5}{|l|}{ Site of extrahepatic metastasis } \\
\hline Lungs & $0.92(0.74-1.14)$ & 0.440 & & \\
\hline Bones & $1.22(0.99-1.52)$ & 0.065 & & \\
\hline Lymph nodes & $0.96(0.74-1.24)$ & 0.740 & & \\
\hline
\end{tabular}

${ }^{a}$ Tumor-node-metastasis classification. HCC, hepatocellular carcinoma; HR, hazard ratio; CI, confidence interval; HCV, hepatitis C virus; AST, aspartate aminotransferase; WBC, white blood cell; NLR, neutrophil-to-lymphocyte ratio; PLR, platelet-to-lymphocyte ratio; GPS, Glasgow prognostic score; AFP, $\alpha$-fetoprotein; DCP, des-gamma-carboxyprothrombin.

HCC diagnosis and extrahepatic lesions. HCC was diagnosed based on high signal intensity images in the hepatic arterial-dominant phase and wash-out images in the venous and delayed phases by either contrast-enhanced computed tomography $(\mathrm{CT})$ or magnetic resonance imaging (MRI), which were then radiographically confirmed. We also measured the serum concentrations of $\alpha$-fetoprotein (AFP) and des-gamma-carboxy prothrombin (DCP). The tumors were biopsied if the imaging findings were inconsistent with characteristics specific to HCC, or if we did not observe an elevation in tumor marker levels. Pulmonary lesions were detected by X-ray or CT of the chest to assess extrahepatic metastasis. These examinations were conducted routinely on the first hospital visit and once every 3-6 months during the follow-up period. Additional examinations, such as bone scintigraphy and CT or MRI of the brain, were conducted in the event of symptoms suspicious of extrahepatic metastasis. These additional examinations were also performed if elevated serum concentrations of AFP and/or DCP were detected, and the elevation could not be explained by the status of the intrahepatic lesion. Positron CT was also performed as a supplementary examination.

Follow-up and endpoints. Each patient was closely followed up, in terms of monitoring intrahepatic lesions and investigating the presence of extrahepatic metastasis following HCC diagnosis. Serum biochemical tests were performed and AFP and DCP levels were measured. Furthermore, ultrasound examinations were performed once every 1-2 months, contrast-enhanced CT and/or MRI were performed once every 2-6 months, and tests using other diagnostic imaging modalities were performed as required. The endpoints used in the present study were the date of death and the date of the final follow-up. The present study was completed in September, 2013 and the median follow-up period was 5.7 months (range, 0.2-111.9 months).

Statistical analysis. Continuous variables are expressed as median values (range). Overall survival was determined by Kaplan-Meier analysis and differences between subgroups were compared with log-rank tests. A Cox proportional hazards stepwise model was used for univariate and multivariate analysis to identify any independent variables related to overall survival. Data from these models are expressed as hazard ratios (HR) and 95\% confidence intervals (95\% CI). All $\mathrm{P}$-values were two-tailed and values $<0.05$ were considered to indicate statistically significant differences. Statistical analysis was performed by SPSS software, version 20 (IBM SPSS, Armonk, NY, USA).

\section{Results}

Overall survival. The overall median survival time (MST) was 7.3 months. The survival rates at 1,2, 3 and 5 years were $31.8,14.5,7.7$ and $0.7 \%$, respectively.

Prognosis analysis results. The results of our prognosis analysis are presented in Table II. As a result of the univariate analysis, 
A

GPS

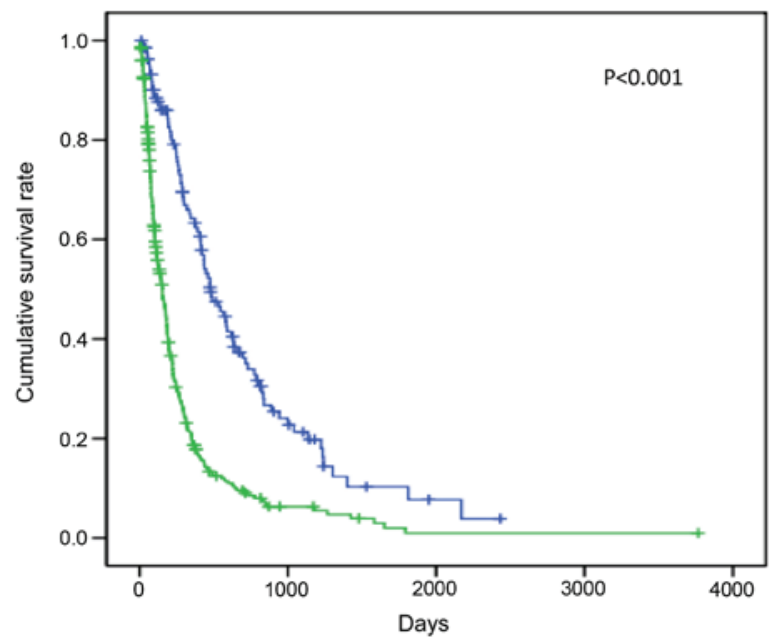

B

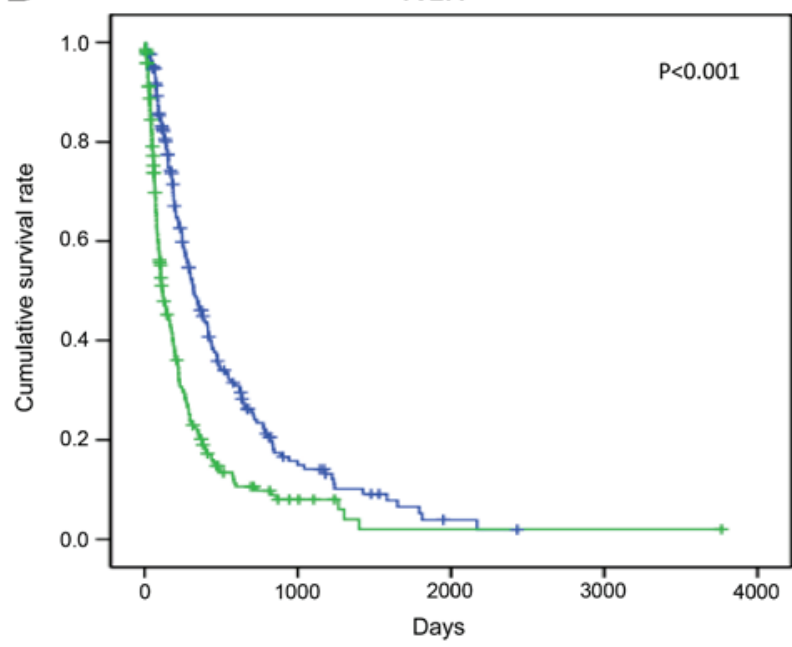

Figure 1. Stratified cumulative survival for hepatocellular carcinoma patients diagnosed with extrahepatic metastases based on the (A) Glasgow prognostic score (GPS) and (B) neutrophil-to-lymphocyte ratio (NLR).

we observed that Child-Pugh class $(\mathrm{B}+\mathrm{C})$, alanine aminotransferase ( $\geq 80 \mathrm{U} / 1)$, WBC count $(\geq 6,000 / 1)$, NLR $(\geq 3)$, PLR $(\geq 111)$, AFP $(\geq 200 \mathrm{ng} / \mathrm{ml})$ and tumor stage $(\mathrm{T} \geq 3)$ were significant risk factors that were adversely associated with survival. The multivariate analysis revealed that hepatic functional reserve (Child-Pugh class $\mathrm{B}+\mathrm{C}$; HR=2.055; 95\% CI: 1.592-2.651, $\mathrm{P}<0.001)$, $\mathrm{T}$ stage $(\mathrm{T} \geq 3 ; \mathrm{HR}=2.359 ; 95 \% \mathrm{CI}: 1.648-3.376$, $\mathrm{P}<0.001)$, AFP $(\geq 200 \mathrm{ng} / \mathrm{ml} ; \mathrm{HR}=1.416 ; 95 \%$ CI: $1.125-1.783$, $\mathrm{P}=0.003), \operatorname{NLR}(\geq 3 ; \mathrm{HR}=1.569 ; 95 \% \mathrm{CI}: 1.253-1.963, \mathrm{P}<0.001)$ and GPS $(1+2 ; \mathrm{HR}=1.410 ; 95 \% \mathrm{CI}: 1.060-1.874, \mathrm{P}=0.018)$ were independent predictive factors of survival.

Correlation of GPS and NLR scores with survival. The cumulative survival curves for GPS and NLR are shown in Fig. 1. A total of 136 patients were included in the GPS 0 group, 169 in the GPS 1 group and 129 in the GPS 2 group; the low together with the high NLR groups included 217 patients. The MST was 480 days in the GPS 0 group, 154 days in the GPS 1 and 2 groups, 115 days in the high NLR group and 321 days in the low NLR group; a significant difference in survival was observed for the GPS and NLR groups.

\section{Discussion}

As regards clinical characteristics, including main site of metastasis and survival, our results were similar to those of previous reports (17-22). The analysis of prognosis revealed that the degree of intrahepatic tumor progression ( $\mathrm{T}$ stage), hepatic functional reserve (Child-Pugh class) and tumor marker levels (AFP) were all significant prognostic factors, as previously reported $(19,21,22)$. In the present study, we observed that, of the SIR indices, GPS and NLR were useful prognostic factors. It has been reported that common sites of extrahepatic metastasis that are clinically characteristic for this stage of HCC in patients aged $>50$ years include the lungs (39.5-67.3\%), followed by the lymph nodes (27.9-45.0\%) and bone (18.3-35.8\%) (17-22). As regards prognosis, the MST has been reported to be 4.9-8.1 months $(19,20,22)$ whereas survival analyses indicate that the state of the intrahepatic tumor,
Child-Pugh class and tumor markers are independent prognostic factors of survival $(19,20,23)$. The analysis of stage IVB HCC prognosis indicates that there are more deaths from intrahepatic events rather than from extrahepatic events, and the degree of intrahepatic tumor progression is a significant risk factor (20). Furthermore, it has been reported that AFP is a marker of tumor progression (22), and that total bilirubin, Alb and Child-Pugh class are indices of hepatic functional reserve $(19,22,24)$.

The present study demonstrated anew that NLR and GPS are useful prognostic factors in patients with stage IVB HCC. It has recently been reported that various markers used in the detection of SIR, such as cytokines, CRP and absolute neutrophil and lymphocyte counts and their ratios (including NLR), play a useful prognostic role in cancer. Of those, NLR is one of the simplest and most effective inflammatory markers, and is associated with a poor prognosis in various types of cancer (25-27). A meta-analysis of the usefulness of NLR as a prognostic factor in HCC was recently published (28). The results indicated that the NLR cut-off value varied according to various treatments, such as transplantation, hepatectomy, RFA and TACE. However, in terms of survival in all treatment groups, a high NLR was strongly correlated with poor prognosis.

It remains unclear why a high NLR is associated with poor prognosis. In general, cancer is considered to cause abnormalities in the differential WBC count via cytokines. A high NLR is observed when the absolute neutrophil count is high and the absolute lymphocyte count is low. It has been reported that the mechanism causing neutrophilia involves a systemic/localized inflammatory response to a tumor or virus that leads to microcirculatory changes favorable for cancer progression or metastasis. It has also been suggested that a high NLR correlates with the strong invasion and activation of tumor-related macrophages (TAM) within the tumor, which promotes neutrophilia via cytokines such as interleukin (IL)-6 and IL-8 (28). It has also been demonstrated that neutrophils produce matrix metalloproteinase 9 , which is involved in angiogenesis, thus promoting the development of tumors and 
chemokines, and vascular endothelial growth factor, thereby promoting tumor development and metastasis (29-31). In accordance, an increase in neutrophils leads to an increase in angiogenic growth factors and precipitates circulatory changes beneficial for the development of HCC; as a result, it increases the likelihood of extrahepatic metastasis and decreases HCC patient survival.

The mechanism underlying the decrease in the lymphocyte count involves the activation of TAM, which leads to the release of immunosuppressive cytokines (IL-10 and transforming growth factor- $\beta$ ) and the decrease in cytotoxic $\mathrm{T}$ lymphocytes. The relative lymphocyte count and $\mathrm{T} 4 / \mathrm{T} 8$ ratio tend to decrease, resulting in a weak inflammatory response via lymphocytes in patients with a high NLR (32).

At present, a proposed hypothesis for the aforementioned correlation between high NLR and poor prognosis in stage IVB HCC patients is that inflammatory markers significantly promote cytokines associated with the development of advance-staged cancer. The mechanisms underlying metastasis also involve various cytokines. It is hypothesized that cytokines are involved in the progression of the underlying liver disease. Thus, inflammatory markers, such as NLR, are considered to be useful for advanced cancer with metastasis that develops on a background of chronic liver disease.

McMillan (33) proposed GPS as an index of nutritional status based on CRP and the Alb-induced inflammatory response. In recent years, the deterioration of nutritional status and chronic inflammatory response have been found to be involved in the status and progression of cancer. Several reports indicate that the GPS is correlated with treatment outcomes and prognosis of various cancers when scored on the basis of the Alb level, which is an indicator of nutritional status, and the inflammatory CRP level (33). The GPS scores are classified in relation to a CRP level of $1.0 \mathrm{mg} / \mathrm{dl}$ and an Alb level of $3.5 \mathrm{~g} / \mathrm{dl}$ as follows: CRP $\leq 1.0 \mathrm{mg} / \mathrm{dl}$ with $\mathrm{Alb} \geq 3.5 \mathrm{~g} / \mathrm{dl}$ is scored as 0 points (normal); $\mathrm{CRP} \leq 1.0 \mathrm{mg} / \mathrm{dl}$ with $\mathrm{Alb}<3.5 \mathrm{~g} / \mathrm{dl}$ is scored as 1 point (malnutrition); CRP $>1.0 \mathrm{mg} / \mathrm{dl}$ with $\mathrm{Alb} \geq 3.5 \mathrm{~g} / \mathrm{dl}$ is scored as 1 point (pre-cancer cachexia); and CRP $>1.0 \mathrm{mg} / \mathrm{dl}$ with Alb $<3.5 \mathrm{~g} / \mathrm{dl}$ is scored as 2 points (cancer cachexia). The higher the GPS score, the stronger the correlation with poor treatment outcome and prognosis. It has been reported that this score is strongly correlated with treatment outcome and prognosis in HCC (33). Cachexia is reported in 20-80\% of cancer patients, and is significantly correlated with the patient's quality of life and prognosis. This score is even more useful due to the high incidence of cachexia in gastrointestinal cancer.

In conclusion, we consider the inflammatory markers GPS and NLR to be useful prognostic factors in patients with stage IVB HCC. However, while the inflammatory response described herein may be very useful for simple, low-cost general screening, its clinical application may be problematic. The inflammatory response may be biased by infection and stress not asociated with cancer. Therefore, the establishment of a useful cut-off value in clinical practice remains a limitation that requires further investigation.

\section{References}

1. Parkin DM, Bray F, Ferlay J and Pisani P: Global cancer statistics, 2002. CA Cancer J Clin 55: 74-108, 2005.
2. Portolani N, Coniglio A, Ghidoni S, Giovanelli M, Benetti A, Tiberio GA and Giulini SM: Early and late recurrence after liver resection for hepatocellular carcinoma: Prognostic and therapeutic implications. Ann Surg 243: 229-235, 2006.

3. Yang Y, Nagano H, Ota H, Morimoto O, Nakamura M, Wada H, Noda T, Damdinsuren B, Marubashi S, Miyamoto A, et al: Patterns and clinicopathologic features of extrahepatic recurrence of hepatocellular carcinoma after curative resection. Surgery 141: 196-202, 2007.

4. Llovet JM, Ricci S, Mazzaferro V, Hilgard P, Gane E, Blanc JF, de Oliveira AC, Santoro A, Raoul JL, Forner A, et al: Sorafenib in advanced hepatocellular carcinoma. N Engl J Med 359: 378-390, 2008.

5. Cheng AL, Kang YK, Chen Z, Tsao CJ, Qin S, Kim JS, Luo R, Feng J, Ye S, Yang TS, et al: Efficacy and safety of sorafenib in patients in the Asia-Pacific region with advanced hepatocellular carcinoma: A phase III randomised, double-blind, placebo-controlled trial. Lancet Oncol 10: 25-34, 2009.

6. Bruix J and Sherman M; American Association for the Study of Liver Diseases: Management of hepatocellular carcinoma: An update. Hepatology 53: 1020-1022, 2011.

7. Kudo M, Izumi N, Kokudo N, Matsui O, Sakamoto M, Nakashima O, Kojiro M and Makuuchi M; HCC Expert Panel of Japan Society of Hepatology: Management of hepatocellular carcinoma in Japan: Consensus-based clinical practice guidelines proposed by the Japan Society of Hepatology (JSH) 2010 updated version. Dig Dis 29: 339-364, 2011.

8. Forrest LM, McMillan DC, McArdle CS, Angerson WJ and Dunlop DJ: Evaluation of cumulative prognostic scores based on the systemic inflammatory response in patients with inoperable non-small-cell lung cancer. Br J Cancer 89, 1028-1030, 2003.

9. Gomez D, Farid S, Malik HZ, Young AL, Toogood GJ, Lodge JP and Prasad KR: Preoperative neutrophil-to-lymphocyte ratio as a prognostic predictor after curative resection for hepatocellular carcinoma. World J Surg 32: 1757-1762, 2008.

10. Huang ZL, Luo J, Chen MS, Li JQ and Shi M: Blood neutrophil-to-lymphocyte ratio predicts survival in patients with unresectable hepatocellular carcinoma undergoing transarterial chemoembolization. J Vasc Interv Radiol 22: 702-709, 2011.

11. Halazun KJ, Hardy MA, Rana AA, Woodland DC IV, Luyten EJ, Mahadev S, Witkowski P, Siegel AB, Brown RS Jr and Emond JC: Negative impact of neutrophil-lymphocyte ratio on outcome after liver transplantation for hepatocellular carcinoma. Ann Surg 250: 141-51, 2009.

12. Bertuzzo VR, Cescon M, Ravaioli M, Grazi GL, Ercolani G, Del Gaudio M, Cucchetti A, D'Errico-Grigioni A, Golfieri R and Pinna AD: Analysis of factors affecting recurrence of hepatocellular carcinoma after liver transplantation with a special focus on inflammation markers. Transplantation 91: 1279-1285, 2011.

13. Dan J, Zhang Y, Peng Z, Huang J, Gao H, Xu L and Chen M: Postoperative neutrophil-to-lymphocyte ratio change predicts survival of patients with small hepatocellular carcinoma undergoing radiofrequency ablation. PLoS One 8: e58184, 2013.

14. Mantovani A, Allavena P, Sica A and Balkwill F: Cancer-related inflammation. Nature 454: 436-444, 2008.

15. Trinchieri G: Cancer and inflammation: an old intuition with rapidly evolving new concepts. Annu Rev Immunol 30: 677-706, 2012.

16. Pawlik TM, Esnaola NF and Vauthey JN: Surgical treatment of hepatocellular carcinoma: Similar long-term results despite geographic variations. Liver Transpl 10 (2 Suppl 1): S74-S80, 2004.

17. Yoo DJ, Kim KM, Jin YJ, Shim JH, Ko GY, Yoon HK, Sung KB, Lee JL, Kang YK, Lim YS, et al: Clinical outcome of 251 patients with extrahepatic metastasis at initial diagnosis of HCC: Does transarterial chemoembolization improve survival in these patients? J Gastroentel Hepatol 26: 145-154, 2011.

18. Katyal S, Oliver JH III, Peterson MS, Ferris JV, Carr BS and Baron RL: Extrahepatic metastases of hepatocellular carcinoma. Radiology 216: 698-703, 2000.

19. Uka K, Aikata H, Takaki S, Shirakawa H, Jeong SC, Yamashina K, Hiramatsu A, Kodama H, Takahashi S and Chayama K: Clinical features and prognosis of patients with extrahepatic metastases from hepatocellular carcinoma. World J Gastroenterol 13: 414-420, 2007.

20. Uchino K, Tateishi R, Shiina S, Kanda M, Masuzaki R, Kondo Y, Goto T, Omata M, Yoshida H and Koike K: Hepatocellular carcinoma with extrahepatic metastasis: Clinical features and prognostic factors. Cancer 117: 4475-4483, 2011. 
21. Ishii H, Furuse J, Kinoshita T, Konishi M, Nakagohri T, Takahashi S, Gotohda N, Nakachi K and Yoshino M: Extrahepatic spread from HCC: Who are candidates for aggressive anti-cancer treatment? Jpn J Clin Oncolol 34: 733-739, 2004

22. Natsuizaka M, Omura T, Akaike T, Kuwata Y, Yamazaki K, Sato T, Karino Y, Toyota J, Suga T and Asaka M: Clinical features of hepatocellular carcinoma with extrahepatic metastases. J Gastroenterol Hepatol 20: 1781-1787, 2005

23. Yoo DJ, Kim KM, Jin YJ, Shim JH, Ko GY, Yoon HK, Sung KB, Lee JL, Kang YK, Lim YS, et al: Clinical outcome of 251 patients with extrahepatic metastasis at initial diagnosis of hepatocellular carcinoma: Does transarterial chemoembolization improve survival in these patients? J Gastroenterol Hepatol 26: 145-154, 2011.

24. Pawarode A, Voravud N, Sriuranpong V, Kullavanijaya P and Patt YZ: Natural history of untreated primary HCC: A retrospective study of 157 patients. Am J Clin Oncol 21: 386-391, 1998.

25. Ding PR, An X, Zhang RX, Fang YJ, Li LR, Chen G, Wu XJ, Lu ZH, Lin JZ, Kong LH, et al: Elevated preoperative neutrophil to lymphocyte ratio predicts risk of recurrence following curative resection for stage IIA colon cancer. Int J Colorectal Dis 25: $1427-1433,2010$

26. Jung MR, Park YK, Jeong O, Seon JW, Ryu SY, Kim DY and Kim YJ: Elevated preoperative neutrophil to lymphocyte ratio predicts poor survival following resection in late stage gastric cancer. J Surg Oncol 104: 504-510, 2011.
27. Stotz M, Gerger A, Eisner F, Szkandera J, Loibner H, Ress AL, Kornprat P, AlZoughbi W, Seggewies FS, Lackner C, et al: Increased neutrophil-lymphocyte ratio is a poor prognostic factor in patients with primary operable and inoperable pancreatic cancer. Br J Cancer 109: 416-321, 2013.

28. Xiao WK, Chen D, Li SQ, Fu SJ, Peng BG and Liang LJ: Prognostic significance of neutrophil-lymphocyte ratio in hepatocellular carcinoma: A meta-analysis. BMC Cancer 14: 117, 2014.

29. Coussens LM, Tinkle CL, Hanahan D and Werb Z: MMP-9 supplied by bone marrow-derived cells contributes to skin carcinogenesis. Cell 103: 481-490, 2000.

30. Strieter RM, Burdick MD, Mestas J, Gomperts B, Keane MP and Belperio JA: Cancer CXC chemokine networks and tumour angiogenesis. Eur J Cancer 42: 768-778, 2006.

31. Gong Y and Koh DR: Neutrophils promote inflammatory angiogenesis via release of preformed VEGF in an in vivo corneal model. Cell Tissue Res 339: 437-448, 2010.

32. Chew V, Tow C, Teo M, Wong HL, Chan J, Gehring A, Loh M, Bolze A, Quek R, Lee VK, et al: Inflammatory tumour microenvironment is associated with superior survival in hepatocellular carcinoma patients. J Hepatol 52: 370-379, 2010.

33. McMillan DC: The systemic inflammation-based Glasgow prognostic score: A decade of experience in patients with cancer. Cancer Treat Rev 39: 534-540, 2013 